\title{
Wittgenstein und Sraffa Zeitproduktion durch Zeit
}

\author{
Peter Weibel, Karlsruhe
}

In seinem berühmten Tractatus Logico-Philosophicus (1921) präsentierte Ludwig Wittgenstein seine Bildtheorie der Sprache: „Der Satz ist ein Bild der Wirklichkeit“ (Tractatus Logico-Philosophicus, 4.01). Sprache, wenn sie adäquat benutzt wird, bildet die Realität ab. Daher ist es die Herausforderung der Philosophie, die Sprache zu entwirren, um Probleme zu klären. Die kritische Betrachtung der Sprache ist das Ziel des Philosophen. Zwischen Sprache und Realität besteht eine Verbindung, eine gemeinsame logische Form. Alles was ist kann in einer formalen Sprache ausgedrückt und beschrieben werden. Was nicht gesagt werden kann, darüber sollte man schweigen.

Während seiner Zeit in Cambridge modifizierte Wittgenstein seine Bildtheorie radikal. Daher wird auch gerne von Wittgenstein I und Wittgenstein II gesprochen. Kurz gesagt kann man von der Bildtheorie der Sprache (Wittgenstein I) und der Theorie des Sprachspiels (Wittgenstein II) sprechen, wie am besten in Wittgensteins Philosophischen Untersuchungen (Philosophical Investigations) beschrieben. Doch wie ist Wittgensteins radikale Abkehr von seiner zuerst aufgestellten Theorie der Sprache zu erklären? In seiner Theorie des Sprachspiels wird Bedeutung nicht abgeleitet von der logischen Form wie in der Bildtheorie der Sprache, sondern es wird davon ausgegangen, dass die Bedeutung eines Satzes allein durch seinen Gebrauch hergestellt wird. Der Gebrauch gibt den Worten eine Bedeutung, nicht ihre logische Form. Im Vorwort zu den Philosophischen Untersuchungen gibt Wittgenstein einen Hinweis darauf, wie er zu dieser neuen Sicht der Dinge gelangte: „Mehr noch als dieser - stets kraftvollen und sicheren Kritik [von Frank Ramsey] verdanke ich derjenigen, die ein Lehrer dieser Universität, Herr P. Sraffa, durch viele Jahre unablessig [sic!] an meinen Gedanken geübt hat." Wer war dieser Piero Sraffa, der so nachhaltigen Einfluss auf Wittgenstein hatte? Sraffa war ein enger Freund des italienischen revolutionären Marxisten Antonio Gramsci. Während Gramsci, von Mussolini inhaftiert, im Gefängnis saß, versorgte Sraffa ihn mit Büchern, Schreibutensilien etc. und blieb in engem Kontakt mit ihm. Unter anderem von Gramscis Philosophie der Tat beeinflusst, schrieb er einen kritischen Artikel im Manchester 
Guardian, der Mussolini verärgerte und Sraffa gefährdete. Aufgrund dessen lud John Maynard Keynes Sraffa ein, an das King's College nach Cambridge zu kommen. Sraffa wurde dort Bibliothekar und später Fellow am Trinity College. Schnell wurde er ein wichtiges Mitglied der akademischen Welt - er gehörte zu der so genannten „Cafeteria Gruppe“, der sich auch Frank Ramsey und Ludwig Wittgenstein anschlossen und die das von J.M. Keynes 1921 aufgestellten Traktat über Wahrscheinlichkeitstheorie (Treatise on Probability) untersuchten. Zusammen mit Keynes versuchte Sraffa die Theorien von Friedrich Hayek zu widerlegen (Sraffa, 1932). Ab 1931 begann er zudem, die Schriften von David Ricardo (Nationalökonom, 1772-1823) zu sammeln und zu editieren, die 1953 publiziert wurden. Seine Einführung in das Buch ist bemerkenswert und gab der Neoricardianischen Schule und der Kapitalkontroverse (Cambridge-Capital-Controversy) einen großen Aufschwung. Doch vor allem Sraffas Buch Production of Commodities by Means of Commodities: Prelude to a Critique of Economic Theory (Warenproduktion mittels Waren), das er in den 1920er Jahren begonnen hatte und das 1960 publiziert wurde, bestätigt Ricardos Theorie für die Moderne.

Sraffas Einfluss auf Wittgenstein kann offensichtlich als Übertragungsfunktion betrachtet werden. Sraffas Ansichten stammen von einer Philosophie der Aktion, die Gramsci prägte und die auch Wittgenstein beeindruckte. Indem Sraffa mit Gramsci und später mit Wittgenstein diskutierte, waren es Gramscis Ideen, die, durch Sraffa gefiltert, Wittgensteins Sicht der Welt prägten. Die Wendung von der Bildtheorie der Sprache hin zur Theorie des Sprachspiels geht also auf Sraffa und Gramsci zurück. Wittgenstein war generell in politischen und ideologischen Dingen sehr konservativ gewesen, doch in seiner späten Theorie der Sprache kam er zu einer sehr demokratischen, um nicht zu sagen marxistischen Sicht der Dinge. Sraffa selbst ging über Gramscis Theorie hinaus und verfasste seine eigene ökonomische Theorie. Man kann drei Entwicklungsstufen erkennen:

1. Ricardo definierte als Ware von allem die Arbeit. Arbeit wiederum definiert den Wert und Preis einer Ware.

2. Marx definierte den Wert und Preis einer Ware ähnlich wie Ricardo, jedoch etwas präziser, da er eine neue Kategorie einführte: die Zeit - und zwar im Sinne der Arbeitszeit. Diese neue Kategorie ermöglichte es ihm, seine Theorie über den Mehrwert aufzustellen.

3. Sraffa vernachlässigte die Kategorie Arbeit als Quelle für den Wert einer Ware. Er schlug eine Input-Output-Analyse der Waren vor, die 
dann den Wert der Waren bestimmen sollte. Die Frage ist also, wie viele Waren braucht man, um eine andere Ware produzieren zu können. Wie viele Waren muss man produzieren, um eine andere Ware konsumieren zu können. Es gibt zwei Möglichkeiten, wie Mehrwert (den der Kapitalist auf dem Rücken der Arbeiter austrägt) produziert werden kann. Der Arbeitstag, also die Arbeitszeit, kann verlängert werden, was Marx als absoluten Mehrwert definierte, oder technische Innovationen können die Arbeitszeit für einen Arbeitsprozess verkürzen - ein relativer Mehrwert. In jedem Fall hat man eine Gleichung, in der die Zeit eine Rolle spielt. Entweder die Arbeitszeit ist länger, damit mehr Güter produziert werden können, während der Lohn gleich bleibt; so entsteht ein Mehrwert für den Kapitalisten, der die Produktionsmittel besitzt. Oder die Arbeitszeit wird aufgrund von technischen Innovationen kürzer und die Löhne werden aufgrund der kürzeren Arbeitszeiten gesenkt, ohne dass die Produktion weniger wird; so entsteht der Mehrwert für den Kapitalisten. Man kann also sagen, Arbeit ist produktiv, wenn sie relativen Mehrwert produziert. Genauer, die Arbeitszeit ist die Quelle für den Mehrwert. Das Zeitverhältnis ist die Quelle für den Mehrwert.

Diese Betrachtung verändert unsere Einstellung bezüglich des Konsums. In der klassischen Wirtschaftstheorie wurde der Konsum als unproduktiv betrachtet, da kein Mehrwert erzeugt wurde - im Gegenteil, der Konsument „verlor“ dadurch sogar Geld. Wenn man die Produktivität mit dem Mehrwert verknüpft, erscheint der Konsum, da hier kein Mehrwert produziert wird, als unproduktiv. Verknüpft man aber die Produktivität nicht mit dem Mehrwert, sondern sieht Produktion und Konsumption als separate Kategorien, kann man folgendermaßen abstrahieren: es gibt eine Produktionszeit und eine Zeit des Konsums. In beiden Fällen haben wir es mit bestimmten Zeitverhältnissen zu tun. Wenn man nicht genug Produktionszeit produziert (äquivalent zum Geld, das man bekommt), hat man nicht genug Zeit für den Konsum (äquivalent zum Geld, das man ausgeben kann). Daher muss man zu Marx Ausspruch „Geld als Wertmaß ist notwendige Erscheinungsform des immanenten Wertmaßes der Ware, der Arbeitszeit“ (Marx 1962, S. 109) hinzufügen: die Maßeinheit für Wert ist Zeit, und zwar Arbeits- und Konsumptionszeit. Arbeit und Konsum sind Vektoren der Zeit. Aber der Wert der Zeit ist variabel. Für den ungelernten Arbeiter oder Experten auf seinem Gebiet hat Zeit einen 
anderen Wert, was sich in einem unterschiedlichen Preis niederschlägt. Wie werden also Werte zu Preisen? Das berühmte Transformationsproblem der Ökonomie wird durch den Faktor Zeit gelöst. Zeit kann als Geld, in Zahlen und Ziffern ausgedrückt werden. Ricardo definierte Arbeit als Wert und Zeit als dessen Maßeinheit. Der Preis ist die Zeitform des Wertes, und Geld ist die Wertform der Zeit. Daher können Werte in Preise transformiert werden bzw. Wertformen in allgemeine Geldware.

Zeit als Maßeinheit der Arbeit wird zum alleinigen Wertmaß. Der homo economicus ist ein Mensch der Zeit. Auch der Raum wird dem homo economicus zu Geld, weil der Raum ja nur Statthalter der Zeit ist. In einem Roman von Raymond Chandler sagt der Wirt zu seinem Gast, der zu lange an einem Tisch sitzt, zu lange einen Raum okkupiert und zu wenig konsumiert: „Man, this is money space“. Nach „time is money“ nun auch „space is money“, denn auch der Raum verfällt schon seit langem der Wertform des Geldes.

Die verkaufte Zeit steht über dem Eingang zur Industriegesellschaft. Chronometrie, gemessene Zeit, ist die Voraussetzung für eine Marktökonomie, in der Geld zur höchsten Ware wird. Wenn also Taylor und Gilbreth als Betriebsingenieure Zeitstudien betrieben, dann in der richtigen Einschätzung der Lage, dass Zeit das edelste Metall ist, nämlich Gold, das eigentliche invariante Wertmaß. Die Zeit- und Bewegungsstudien um das Ende des 19. Jahrhunderts begleiten eine Industrialisierung der Zeit, sie beweisen den Beginn einer Herrschaft der Zeit.

Zeitstudien waren Sondierungen des Kapitals, der abstrakten Wertform Geld, sie bereiteten den Boden für den subtilen Kapitalismus der postindustriellen Gesellschaft. Der durch den Tauschwert eingeleitete Abstraktionsprozess, der sukzessiv Gegenstände in Waren und Waren in Zeichen verwandelte, dieser Prozess der Aufösung des Objekts, hat sich im postindustriellen Kapitalismus, der ein Feudalismus der Zeit ist, so zugespitzt, dass das Geld selbst verschwindet, weil es noch zu sehr seine Herkunft vom Objekt, von der Ware verrät. Im Zuge der Temporalisierung des Raumes durch die beschleunigte Maschine wurde auch die Arbeit temporalisiert. Nicht nur die Bewegung wurde vom Raum unabhängig, sondern verabsolutiert und verabschiedet haben sich auch der Wert von der Arbeit und der Preis vom Wert. Das führte zu einer Verselbständigung der Preise und der Geldware, zur Abstraktion der Geldzirkulation, die fast irreal und immateriell wurde.

Aktienmarkt, Börsengeschäfte, Wertpapiere, Termin- und Geldwechselgeschäfte, wo Geld mit Geld getauscht wird und der Profit aus der bloßen 
Zeit-Differenz (!) und nicht mehr aus irgendeiner Form produktiver Arbeit entsteht, belegen diese beschleunigte Abstraktion der Arbeit, welche die Arbeit selbst tilgt. Der reine Geldmarkt löscht das Geld - wie im Raumzeitalter der Raum verschwindet. Das Auftauchen der Scheckkarte, die das Geld in dem Maße verdrängt, dass man in den USA sofort der Kriminalität verdächtigt wird, wenn man mit Geld statt mit einem Scheck bezahlt, bezeugt das Verlöschen des Geldes als Objekt, als Ware zweiten Grades, belegt die Verabsolutierung des Geldes zur Immaterialität. Nach der Arbeit und nach der Zeit hat sich auch das Geld so abstrahiert, dass es nicht nur als Objekt zweiten Grades verschwindet, sondern in einer paradoxen Drehung sogar zum Gradmesser von Armut wird. Wer wirklich reich ist, hat kein Geld mehr, zumindest in der Tasche, sondern eine Scheckkarte. In besseren US-Hotels bekommt man mit Bargeld kein Zimmer, sondern nur mit Scheckkarte. Wer Bargeld hat, ist der Ärmste. Wer Bargeld hat, ist im Zeitalter der Scheckkarte, des temporalisierten Geldes, in Wirklichkeit bar des Geldes. Scheck und Kredit zeigen den Kapitalismus als Geschäft mit der Zeit, als Ergebnis der fortschreitenden Abstraktionen und Verselbständigungsprozesse von Arbeit, Wert, Zeit, Geld als die Parameter und Stützen unserer Ökonomie. Dieser vom Tauschwert verursachte Abstraktionsprozess hat die Werttheorie zur Preistheorie verwandelt. Dieser Prozess hat die Arbeit bis zur Verdrängung abstrahiert, so dass im „ökonomischen Kalkül“ (Charles Bettelheim) eine Verschiebung von der Warenproduktion durch Arbeit zur „Warenproduktion mittels Waren“ (nach der gleichnamigen Publikation von Piero Sraffa, 1960) stattfindet. Die Ökonomie der Preise hat sich in der Tat durch die kapitalistische Produktionsweise und Zirkulation des Kapitals so sehr von der Realität der Arbeit entfernt, dass Sraffa zu einem strukturalen mathematischen Modell greifen musste, um die Marktgesetzlichkeiten erklären zu können. Auch die Ökonomie ereignet sich im mathematischen virtuellen Raum. Unter dem Diktat der Zeit und der Chronometrie wird auch die Ökonomie zur Ökometrie.

Die letzte Stufe der Abstraktion der Arbeit ist die gegenwärtige Zeitproduktion mittels Zeit. Die Ökonomie lebt davon, dass die Arbeitenden ihre (Lebens)Zeit investieren und verkaufen (in Form von Arbeitszeit), um Geld zu verdienen, damit sie ihre Freizeit (in Form von Waren, Erholung und Subsistenzmitteln) kaufen können. Zeit ist es, was investiert, gekauft und verkauft wird. Daher sind die so genannten Zeit- und Termingeschäfte die lukrativsten. Die höchste, abstrakte Form des Kapitalismus ist die Monetarisierung der Zeit. Das ist die logische Konsequenz jener Gleichung, 
die Arbeitszeit und Wertzeit, Zeitwert und Arbeitswert gleichsetzte. Auch der Marxismus hat sich diesem fatalen Horizont nicht entzogen. Die Inflationen und Krisen des Kapitalismus entstehen aus Zeitimplosionen im ökonomischen Kalkül. Benjamin Coriat beschreibt in L'atelier et le chronomètre (1979) den Beginn des Zeitkapitalismus im Fordismus.

Im Zeitalter der Arbeitsökonomie gab es die Angst vor der Überproduktion von Waren. Eine Krise der Überproduktion entstünde, würde nicht gleichzeitig eine Massenkonsumption von Waren eintreten, indem diese billig genug sind, dass die Arbeiter sie kaufen können. Deswegen hat Henry Ford bekanntlich gesagt, unsere Arbeiter sollten auch unsere Kunden sein. In einer Art Selbststeuerung des Marktes waren die Arbeiter in großem Maße auch Konsumenten der Waren, die sie produzierten. Auf dieser Basis der Massenproduktion und Massenkonsumption wurde intensiv Kapital akkumuliert, entstanden die großen Vermögen Amerikas. Diese Selbstregulation geriet aber in eine Krise der Überproduktion, als der wichtigste Markt für solche Massenprodukte, nämlich der Haushalt, gesättigt war. Der zweite Grund war die beschleunigte Produktivität, nämlich die Ersetzung von Menschen durch Maschinen im automatisierten Produktionsprozess, sie hat das Problem der Überproduktion drastisch verschärft. Die durch die Automation arbeitslosen Arbeiter verfügten einerseits über eine Überfülle von Zeit, andererseits über keine Kaufkraft, da es sich um eine Überfülle von Stehzeit handelte. Eine Schere begann sich zu öffnen: die Produktion von Massenwaren beschleunigte sich, aber gleichzeitig reduzierte sich die Masse der Arbeiter, welche die Massengüter kaufen konnten. Ein und dieselbe Quelle, nämlich Maschinen, war sowohl für das Ansteigen der Produktivität wie das Absinken der Kaufkraft verantwortlich. Je mehr durch Maschinen produziert wurde und je mehr Arbeiter durch maschinelle automatisierte Produktion „freigesetzt" wurden, umso mehr Waren gab es, aber ebenso umso weniger Arbeiter, die genug Geld verdienten, um die von den Maschinen produzierten Güter kaufen und konsumieren zu können. Der Widerspruch ist am besten in monetärer Zeit formulierbar. Die Arbeiter waren die Konsumenten der Zeit, die sie produzierten. Als jedoch die $\mathrm{Au}-$ tomation immer mehr Zeit produzierte, hatten die Arbeiter immer weniger Zeit, diese zu konsumieren. Eine Überproduktion nicht nur von Konsumgütern, sondern von Zeit war die Folge. Das Problem des Güterkonsums verdeckt nur das Problem des Zeitkonsums, die Überproduktion von Zeit. Die ökonomische Gleichung Arbeit-Wert-Ware-Zeit-Geld wurde durch den Faktor Zeit, durch die maschinelle Beschleunigung der Zeit, aus dem 
Gleichgewicht gebracht. Das ist das eigentliche Problem heute hinter dem so genannten demographischen Problem, dem Verhältnis von Rentnern und Arbeitenden.

Die Automation hat zuviel Zeit erzeugt, eine Überproduktion von Waren und von Zeit. So hat ein komplexes äquilibristisches Spiel von Arbeitszeit, freigesetzter Zeit, von Freizeit und freier Zeit, von Produktions- und Konsumptionszeit begonnen.

Das neue Ziel des Kapitalismus ist also, ein Maximum an Zeit (Konsum) mit einem Minimum an Zeit (Produktion) zu erzeugen, um der Überproduktion (von Waren und Zeit) zu entgehen. Die Theorie des „menschlichen Kapitals“, die jedes Individuum zum Produzenten (oder Künstler) macht, ist von Gary Becker (Human Capital: A Theoretical and Empirical Approach, 1964) bis zu Joseph Beuys (Kreativität alias menschliche Produktivität als Kapital) die neokonservative Konzeption des homo economicus. Wenn ein Individuum (theoretisch verkürzt) zum Produzenten von Waren wird, wird deren Wert gemessen als Maß der Zeit, den das Individuum damit verbringt, sie zu konsumieren, also als konsumierte Zeit. In einer Konsum orientierten Gesellschaft, deren Vektor der Abbau der Überproduktion ist, ist nicht mehr - wie bei Ricardo - die Arbeit die einzig wirkliche Reichtumsquelle der Nation, sondern der Konsum, zumindest in den neokonservativen Köpfen. Ein perpetuum mobile der Zeit ist der uneinlösbare Kern der neokonservativen ökonomischen Theorie. Das Gespenst der Zeit-Ökonomie heißt Überproduktion von Zeit.

Denn um Zeit zu gewinnen, müssen die Individuen Geld verdienen, da ja Zeit Geld und Geld Zeit ist. Um Geld zu verdienen, müssen die Individuen einen Teil ihrer Zeit der Lohnarbeit widmen. Damit diese Zeit der Lohnarbeit nicht zu lang wird, um Zeit in der Produktion von Waren (Konsumzeit) zu gewinnen, muss das Individuum Maschinen bauen, welche den Produktionsprozess beschleunigen, d.h. Wissen erwerben. Das wiederum kostet Zeit; Maschinen sind also gespeicherte und gesparte Zeit. Aber um diese Zeit zu speichern, um mit den Maschinen die Zeit der Lohnarbeit zu verkürzen, um Zeit zu sparen, durch Maschinen, welche Güter schneller produzieren, muss das Individuum vorher diese Zeit hineinstecken, investieren, also die Zeit der Konsumation verzögern und verkürzen. So entsteht in Wirklichkeit ein neuer Asketismus, ein ungeheurer Zeitdruck. Das Individuum wird nämlich, um ein hohes Einkommen zu erreichen, das ihm scheinbar mehr Güter und Konsumzeit verspricht, den Großteil seiner Zeit dem Geldverdienen widmen, was ihm den Kauf jener Maschinen ermögli- 
chen soll, die Zeit sparen, um mehr Geld zu verdienen. Aber wenn die Zeit des Individuums nichts wert ist, sein Lohn ohnehin zu gering, wird es immer mehr Zeit haben, um immer weniger Güter zu konsumieren. Verlust an Zeit für die einen und Verlust an Waren für die anderen sind das Ergebnis der Schere der Zeit: Die einen, deren Zeit teuer ist, weil Experten, werden immer mehr arbeiten müssen, die anderen, die ungelernten Arbeiter, werden immer weniger arbeiten können. Das ist das wahre Gesicht der Zeitakkumulation statt der Kapitalakkumulation. „Kapitalakkumulation wird für ein Individuum nichts anderes sein als die Vermehrung der Waren, die ihm die Mühen ersparen, sich die Zeit zu nehmen, sie zu konsumieren." (Alliez/ Feher, 1986)

Geld kristallisiert Zeit als Produktionszeit in Form von Bezahlung. Verdientes Geld ist dann gesparte Zeit. In Form von Kapital speichert Geld die Zeit der Warenkonsumation. Das Kapital wird zum Geber, Versorger der Zeit. Die absolute Chronokratie/Herrschaft der Zeit hat also begonnen, ein neuer Feudalismus der Zeit, wo es Zeitsklaven und Zeitherren gibt, aneinandergekettet im gemeinsamen Wunsch, die Zeit profitabel zu machen.

Wie es im Feudalismus des 11. Jahrhunderts eine Unterernährung der Armen und ein Überfressen der Reichen gab, so im neuen Feudalismus immer mehr Konsumationszeit (die ja gespeichertes gespartes Geld ist) für die Reichen und immer weniger Konsumationszeit für die Armen (was äquivalent ist mit „freier Zeit“, frei gesetzter Zeit). Wer also keine Zeit hat im Sinne von Zeit als gespartes Geld, als Kaufkraft, muss sich Zeit kaufen. Aber wie, wenn er kein Geld hat, sich Zeit zu kaufen und sein Wunsch nach Zeit ja gerade daher rührt, dass er kein Geld hat? Indem er eben seine Zeit verkauft. Aber wie, wo wir doch davon ausgegangen sind, dass er keine Zeit hat? Im Zeitalter der Raumpolitik konnte man auf Grund und Boden ein Lehen aufnehmen. Im Zeitalter der Chronokratie kann man auf seine Lebenszeit ein Lehen aufnehmen. Das Individuum, dessen gegenwärtige Zeit nicht ausreicht, verkauft seine künftige Zeit, es nimmt ein Lehen auf seine künftige, erst in Zukunft sich kapitalisierende Zeit; es nimmt ein Dar-Lehen, einen Kredit auf. Es tauscht mit Zeit. Es kauft sich Konsumationszeit, damit es sich jetzt schon die Güter kaufen kann, für die es noch nicht genügend Geld (Zeit) produziert hat und die es jetzt konsumieren will, indem es künftige Zeit verkauft. Dafür muss dieser Zeitknecht natürlich Zinsen zahlen, Zeitzinsen. Er muss nämlich zumeist ein Drittel mehr an Zeit in Zukunft investieren, produzieren, bezahlen, was die Ware jetzt an Zeit gekostet hat. Das Individuum verliert also Zeit, Lebenszeit bei diesem Zeittausch in 
Form eines Darlehens. Du leihst dir Zeit von der Bank, du nimmst ein Lehen auf deine Lebenszeit bei der Bank auf - die sich wiederum gegen dein frühzeitiges Ableben, dein Ableben vor der Zeit, versichert, was auch du wiederum bezahlen musst -, damit es dir ermöglicht wird, jetzt schon Produkte anzukaufen, die du dir noch nicht leisten kannst, weil du dafür noch nicht genug gearbeitet hast, Geld verdient hast, Zeit produziert hast.

Leih- und Lehn-Zeit sind gewissermaßen simulierte Zeit, Falschzeit. Der eigentliche Lohn der Arbeit heute ist Lehn-Zeit. Aus der Tatsache, dass heute fast alle, Privatpersonen wie Betriebe, von Krediten, Darlehen leben, erkennen wir, in welchem Maße wir schon in einer Chronokratie leben. Nicht mehr die Gegenwart genügt in dieser Wirtschaft, um die notwendige Zeit zu produzieren; alle brauchen mehr Zeit als sie haben. Daher wird auf Kosten künftiger Lebenszeit, auch künftiger Generationen, Zeit für den gegenwärtigen Verbrauch gekauft und geborgt. Alle machen Schulden auf ihre Lebenszeit und die Zeit ihrer Nachfahren. Surrogat-Zeit beherrscht die Ökonomie. Schulden auf Zeit bedeutet verschuldete Zeit als neues inflationäres Virus der Weltökonomie, die eine Zeitökonomie ist.

\section{Literatur}

Eric Alliez, Michael Feher 1986 „The Luster of Capital“, in: Zone 1/2, New York, 352.

Becker, Gary 1964 Human Capital: A Theoretical and Empirical Approach, New York: Nat. Bureau of Economic Research.

Coriat, Benjamin 1979 L'atelier et le chronomètre: essai sur le taylorisme, le fordisme et la production de masse, Paris: Bourgois.

Marx, Karl 1962 Das Kapital, Band 1, Berlin: Dietz.

Sraffa, Piero 1932 „Dr. Hayek on Money and Capital“, in: Economic Journal XLII, 42-53.

- 1960 Production of commodities by means of commodities: prelude to a critique of economic theory, Cambridge: Cambridge Univ. Press.

Wittgenstein, Ludwig 2003 Tractatus Logico-Philosophicus, Frankfurt/M.: Suhrkamp.

- 1971 Philosophische Untersuchungen, Frankfurt/M.: Suhrkamp. 
